\title{
Floods in North-Central and Eastern South Dakota, Spring 1997
}

INTRODUCTION

Heavy precipitation between September 1996 and February 1997 caused record-breaking spring flooding in north-central and eastern South Dakota. Most of the winter precipitation came during severe snowstorms driven by strong winds that created blizzard conditions over much of the area. The potential for extensive flooding was widely recognized and emergency action plans were put into effect in many communities. A relatively slow warming period during March probably kept the severe flooding from reaching more catastrophic proportions.

The 1997 flooding equaled or exceeded the 500-year recurrence interval at two streamflow-gaging stations and the 100-year recurrence interval at five other gaging stations. This followed 1993 and 1995 flooding, when several streamflowgaging stations had peaks exceeding the 100-year recurrence interval. Peak-flow data for the spring of 1997 at selected gaging stations located on South Dakota streams are shown in table 1 . The listed recurrence intervals are estimates based on a limited period of record.

The U.S. Geological Survey (USGS), one of the principal Federal agencies responsible for the collection and interpretation of water-resources data, works with other Federal, State, and local agencies to ensure that accurate and timely data are available for flood warning, damage assessment, and making decisions regarding the public's welfare.

\section{WINTER OF 1996-97}

Precipitation in South Dakota during the winter of 1996-97 was more than double the normal and was the highest on record, according to the State Climatologist (A.R. Bender, written commun., January 1998). During September 1996 through February 1997, Statewide precipitation averaged 10.8 inches, compared to a long-term average of 5.0 inches for September-February. Total snowfall for November 1996 through March 1997 ranged from near 20 inches at several locations in the southwestern part of the State to nearly 100 inches in areas of the east-central part of the State. The heavy snows were accompanied by persistent strong winds and cold temperatures creating blizzard conditions that closed roads, schools, and businesses many times throughout the winter. Livestock and agricultural losses were extensive. The winter storms killed more than 296,000 head of livestock (U.S. Department of Agriculture, Farm Service Agency, written commun., December 1997).

Due to severe winter storms, the Governor declared a State of Emergency on January 10, 1997. The President approved a Disaster Declaration for all counties of South Dakota the same day. As of September 30, 1997, the State had received slightly more than $\$ 314$ million in Federal disaster-relief funds (Federal Emergency Management Agency, written commun., 1997).

In mid-March, estimated snow water content exceeded 4 inches in most of northeastern South Dakota. Areas in the northeastern and east-central parts of the State had snow water content greater than 5 inches (fig. 1).

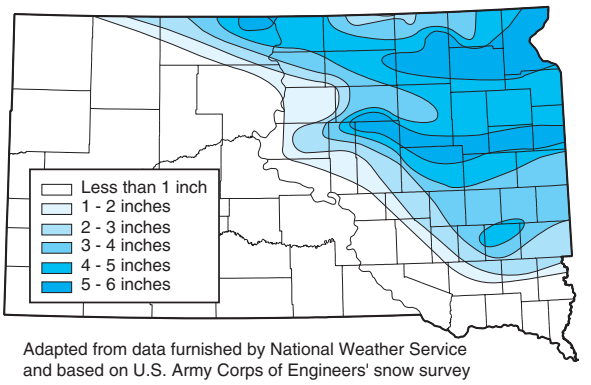

Figure 1. Water content of snow as of March 15-19, 1997.

\section{FLOODS OF 1997}

Ice jams on the White River and some nearby streams caused some flooding in late February in the central part of the State. Flooding began in midMarch in the north-central part of the State when runoff from melting snow

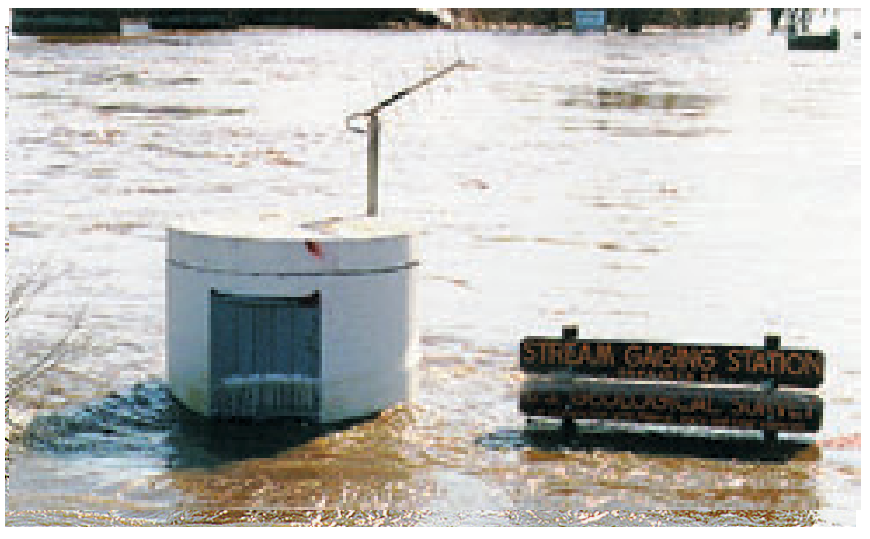

Flooding on the James River at the Huron streamflowgaging station on April 8, 1997.

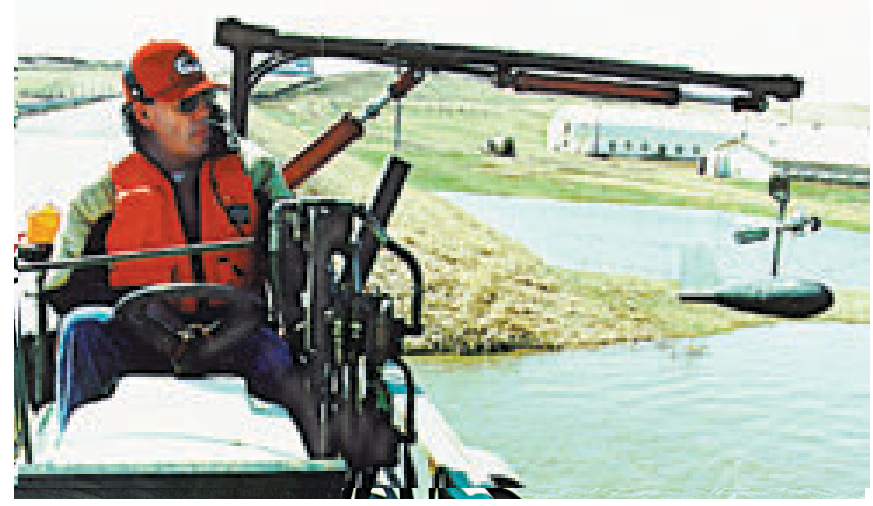

Discharge measurement on the James River near Scotland on April 3, 1997. 
Table 1. Comparison of peak stage and streamflow data for February through May 1997, to previous maximums for period of record at selected gaging stations

$\left[\mathrm{mi}^{2}\right.$, square miles; $\mathrm{ft}$, feet; $\mathrm{ft}^{3} / \mathrm{s}$, cubic feet per second; --, no data, not computed, or not determined; <, less than; >, greater than; BW, backwater; (- ), reverse flow; e, estimated]

\begin{tabular}{|c|c|c|c|c|c|c|c|c|c|c|}
\hline \multirow[b]{2}{*}{$\begin{array}{l}\text { Station } \\
\text { number }\end{array}$} & \multirow[b]{2}{*}{ Station name } & \multirow{2}{*}{$\begin{array}{c}\text { Period } \\
\text { of } \\
\text { record } \\
\text { (water } \\
\text { years) }\end{array}$} & \multirow[b]{2}{*}{$\begin{array}{c}\text { Contributing } \\
\text { drainage } \\
\text { area, } \\
\left(\mathrm{mi}^{2}\right)\end{array}$} & \multicolumn{4}{|c|}{ Flood of February-May 1997} & \multicolumn{3}{|c|}{ Previous maximum } \\
\hline & & & & $\begin{array}{c}\text { Peak } \\
\text { stage } \\
\text { (ft) }\end{array}$ & $\begin{array}{l}\text { Peak } \\
\text { flow } \\
\left(\mathrm{ft}^{3} / \mathrm{s}\right)\end{array}$ & Date & $\begin{array}{c}\text { Recurrence } \\
\text { interval } \\
\text { range }^{1} \\
\text { (years) }\end{array}$ & $\begin{array}{c}\text { Peak } \\
\text { stage } \\
(f t)\end{array}$ & $\begin{array}{l}\text { Peak } \\
\text { flow } \\
\left(\mathrm{ft}^{3} / \mathrm{s}\right)\end{array}$ & Date \\
\hline 05051650 & La Belle Creek near Veblen & $1988-97$ & 8.74 & $\begin{array}{l}12.57 \\
10.60\end{array}$ & $\begin{array}{l}\text { BW } \\
2100\end{array}$ & $\begin{array}{l}4 / 1 \\
4 / 4\end{array}$ & -- & & $\begin{array}{l}\mathrm{BW} \\
664\end{array}$ & $\begin{array}{l}3 / 26 / 89 \\
5 / 18 / 96\end{array}$ \\
\hline 05289985 & Big Coulee Creek near Peever & $1988-97$ & 12.1 & $\begin{array}{l}9.43 \\
7.72\end{array}$ & $\begin{array}{l}\text { BW } \\
358\end{array}$ & $\begin{array}{l}3 / 27 \\
4 / 5\end{array}$ & -- & $\begin{array}{l}9.08 \\
8.21\end{array}$ & $\begin{array}{l}\text { BW } \\
456\end{array}$ & $\begin{array}{l}3 / 11 / 95 \\
6 / 21 / 91\end{array}$ \\
\hline 05292704 & $\begin{array}{l}\text { North Fork Yellow Bank River } \\
\text { near Odessa, MN }\end{array}$ & $1992-97$ & 208 & $\begin{array}{l}18.02 \\
15.94\end{array}$ & $\begin{array}{r}\text { BW } \\
4,760\end{array}$ & $\begin{array}{l}3 / 29 \\
3 / 31\end{array}$ & -- & & 2,580 & $7 / 8 / 94$ \\
\hline 05299700 & Cobb Creek near Gary & 1993-97 & 70.3 & 13.99 & ${ }^{2} 2,100$ & $3 / 31$ & -- & 12.09 & 860 & $6 / 17 / 92$ \\
\hline 06354860 & Spring Creek near Herreid & $\begin{array}{l}1963-86 \\
1989-96\end{array}$ & 220 & 12.64 & 2,680 & $4 / 1$ & $10-25$ & & $\begin{array}{l}1,570 \\
1,630\end{array}$ & $\begin{array}{l}7 / 27 / 93 \\
3 / 15 / 95\end{array}$ \\
\hline 06354882 & Oak Creek near Wakpala & 1985-97 & 356 & 19.62 & 27,500 & $3 / 27$ & $50-100$ & $\begin{array}{l}18.62 \\
17.73\end{array}$ & $\begin{array}{r}\mathrm{BW} \\
3,780\end{array}$ & $\begin{array}{l}3 / 14 / 95 \\
3 / 4 / 86\end{array}$ \\
\hline 06357800 & Grand River at Little Eagle & 1959-97 & 5,370 & $\begin{array}{l}19.58 \\
16.96\end{array}$ & $\begin{array}{r}\text { BW } \\
20,900\end{array}$ & $\begin{array}{l}3 / 24 \\
3 / 27\end{array}$ & $25-50$ & & $\begin{array}{r}\text { BW } \\
31,000\end{array}$ & $\begin{array}{l}3 / 18 / 66 \\
3 / 23 / 87\end{array}$ \\
\hline 06360500 & Moreau River near Whitehorse & $1955-97$ & 4,880 & $\begin{array}{l}27.68 \\
26.93\end{array}$ & $\begin{array}{r}\text { BW } \\
29,700\end{array}$ & $\begin{array}{l}3 / 21 \\
3 / 23\end{array}$ & $25-50$ & $\begin{array}{l}26.20 \\
26.00\end{array}$ & $\begin{array}{r}\text { BW } \\
27,700\end{array}$ & $\begin{array}{l}3 / 14 / 72 \\
5 / 24 / 82\end{array}$ \\
\hline 06442000 & Medicine Knoll near Blunt & 1950-97 & 317 & 13.15 & $\mathrm{e} 4,000$ & $3 / 28$ & $25-50$ & & -- & $6 / 5 / 91$ \\
\hline 06442718 & $\begin{array}{l}\text { Campbell Creek near Lee's } \\
\text { Corner }\end{array}$ & 1988-97 & 54.1 & 14.40 & ${ }^{2} 800$ & $3 / 21$ & -- & 15.01 & 3,260 & $7 / 24 / 93$ \\
\hline 06452000 & White River near Oacoma & 1929-97 & 9,940 & $\begin{array}{l}23.84 \\
--\end{array}$ & $\begin{array}{r}\text { BW } \\
{ }^{2} 29,100\end{array}$ & $\begin{array}{l}2 / 20 \\
2 / 21\end{array}$ & $10-25$ & & $\begin{array}{r}\text { BW } \\
51,900\end{array}$ & $\begin{array}{l}3 / 4 / 94 \\
3 / 30 / 52\end{array}$ \\
\hline 06452320 & Platte Creek near Platte & 1989-97 & 741 & $\begin{array}{r}12.67 \\
9.13\end{array}$ & $\begin{array}{r}\text { BW } \\
1,680\end{array}$ & $\begin{array}{l}5 / 8 \\
2 / 20\end{array}$ & -- & 11.29 & 2,600 & $5 / 11 / 95$ \\
\hline 06471000 & James River at Columbia & & 2,481 & & $\begin{array}{r}\text { BW } \\
24,130 \\
(-2,430\end{array}$ & $\begin{array}{l}4 / 19 \\
4 / 30 \\
3 / 30)\end{array}$ & $25-50$ & & $\begin{array}{r}\text { BW } \\
2,340 \\
(-1,320\end{array}$ & $\begin{array}{l}5 / 13 / 95 \\
5 / 3 / 79 \\
3 / 16 / 95)\end{array}$ \\
\hline 06471200 & $\begin{array}{l}\text { Maple River at North Dakota- } \\
\text { South Dakota State line }\end{array}$ & $1957-97$ & 384 & 16.19 & ${ }^{2} 5,300$ & $3 / 29$ & $25-50$ & 16.05 & ${ }^{2} 5,930$ & $4 / 11 / 69$ \\
\hline 06471500 & Elm River at Westport & $1946-97$ & 1,049 & 21.56 & ${ }^{2} 9,380$ & $3 / 30$ & $10-25$ & & 12,600 & $4 / 10 / 69$ \\
\hline 06472000 & James River near Stratford & $\begin{array}{l}1950-72, \\
1977, \\
1995, \\
1997\end{array}$ & 4,860 & 19.48 & 8,400 & $4 / 6$ & $50-100$ & $\begin{array}{l}19.86 \\
--\end{array}$ & 5,580 & $\begin{array}{l}5 / 18 / 95 \\
5 / 14 / 50\end{array}$ \\
\hline 06473000 & James River at Ashton & & 5,673 & $\begin{array}{l}26.64 \\
25.03\end{array}$ & $\begin{array}{r}\text { BW } \\
29,150 \\
(-8,400\end{array}$ & $\begin{array}{l}4 / 6 \\
4 / 23 \\
3 / 31)\end{array}$ & $>100$ & & $\begin{array}{r}\text { BW } \\
25,680 \\
(-2,100\end{array}$ & $\begin{array}{l}5 / 18 / 95 \\
4 / 24 / 69 \\
4 / 9 / 69)\end{array}$ \\
\hline 06473700 & Snake Creek near Ashton & $\begin{array}{l}1956-72, \\
1977-79, \\
1985-89 \\
1997\end{array}$ & 2,609 & 20.74 & 15,000 & $4 / 1$ & $>100$ & ${ }^{3} 17.21$ & 6,980 & $4 / 10 / 69$ \\
\hline 06474000 & Turtle Creek near Tulare & & 1,124 & $\begin{array}{l}19.32 \\
18.80\end{array}$ & $\begin{array}{r}\text { BW } \\
13,500\end{array}$ & $\begin{array}{l}3 / 26 \\
3 / 28\end{array}$ & $25-50$ & & ${ }^{2} 6,000$ & $4 / 5 / 69$ \\
\hline 06474500 & Turtle Creek at Redfield & $1946-72$ & 1,481 & 18.32 & 13,500 & $3 / 29$ & $50-100$ & 15.94 & 7,660 & $4 / 7 / 69$ \\
\hline 06475000 & James River near Redfield & $1950-97$ & 9,793 & $\begin{array}{l}31.10 \\
29.92\end{array}$ & $\begin{array}{r}\text { BW } \\
17,000\end{array}$ & $\begin{array}{l}4 / 6 \\
4 / 3\end{array}$ & $>100$ & & 9,800 & $5 / 15 / 95$ \\
\hline 06476000 & James River at Huron & $\begin{array}{l}1929-32 \\
1944-97\end{array}$ & 11,721 & 21.28 & 23,400 & $4 / 6$ & $>500$ & 16.86 & 10,000 & $5 / 19 / 95$ \\
\hline 06476500 & Sand Creek near Alpena & & 261 & 13.45 & ${ }^{2} 2,000$ & $3 / 23$ & $10-25$ & & $\begin{array}{r}\mathrm{BW} \\
2,240\end{array}$ & $\begin{array}{l}3 / 28 / 50 \\
3 / 28 / 60\end{array}$ \\
\hline
\end{tabular}


Table 1. Comparison of peak stage and streamflow data for February through May 1997, to previous maximums for period of record at selected gaging stations-Continued

\begin{tabular}{|c|c|c|c|c|c|c|c|c|c|c|}
\hline \multirow[b]{2}{*}{$\begin{array}{c}\text { Station } \\
\text { number }\end{array}$} & \multirow[b]{2}{*}{ Station name } & \multirow{2}{*}{$\begin{array}{c}\text { Period } \\
\text { of } \\
\text { record } \\
\text { (water } \\
\text { years) }\end{array}$} & \multirow[b]{2}{*}{$\begin{array}{l}\text { Contributing } \\
\text { drainage } \\
\text { area, } \\
\left(\mathrm{mi}^{2}\right)\end{array}$} & \multicolumn{4}{|c|}{ Flood of February-May 1997} & \multicolumn{3}{|c|}{ Previous maximum } \\
\hline & & & & $\begin{array}{c}\text { Peak } \\
\text { stage } \\
\text { (ft) }\end{array}$ & $\begin{array}{c}\text { Peak } \\
\text { flow } \\
\left(\mathrm{ft}^{3} / \mathrm{s}\right)\end{array}$ & Date & $\begin{array}{c}\text { Recurrence } \\
\text { interval } \\
\text { range }{ }^{1} \\
\text { (years) }\end{array}$ & $\begin{array}{c}\text { Peak } \\
\text { stage } \\
\text { (ft) }\end{array}$ & $\begin{array}{c}\text { Peak } \\
\text { flow } \\
\left(\mathrm{ft}^{3} / \mathrm{s}\right)\end{array}$ & Date \\
\hline 06477000 & James River near Forestburg & $1950-97$ & 13,442 & 20.61 & 25,600 & $4 / 6$ & $>100$ & $\begin{array}{l}17.26 \\
17.08\end{array}$ & $\begin{array}{r}\text { BW } \\
13,000\end{array}$ & $\begin{array}{l}4 / 22 / 95 \\
5 / 18 / 95\end{array}$ \\
\hline 06477150 & Rock Creek near Fulton & & 240 & 13.74 & 3,120 & $3 / 29$ & $10-25$ & & 2,040 & $4 / 7 / 69$ \\
\hline 06478000 & James River near Mitchell & $\begin{array}{l}1954-58, \\
1966-72, \\
1995 \\
1997\end{array}$ & 14,916 & 23.14 & 28,000 & $4 / 7$ & $50-100$ & 20.43 & 16,200 & $4 / 23 / 95$ \\
\hline 06478500 & James River near Scotland & 1929-97 & 16,505 & 19.87 & 28,000 & $4 / 9$ & $50-100$ & & 29,400 & $6 / 23 / 84$ \\
\hline 06478513 & James River near Yankton & 1982-97 & 16,794 & 22.94 & 28,800 & $4 / 9$ & $10-25$ & 24.34 & 26,400 & $6 / 23 / 84$ \\
\hline 06478535 & $\begin{array}{l}\text { East Fork Vermillion River } \\
\text { near Ramona }\end{array}$ & $\begin{array}{l}1987-89 \\
1996-97\end{array}$ & 508 & 8.50 & 1,600 & $4 / 30$ & -- & & $\begin{array}{l}\text { BW } \\
350\end{array}$ & $\begin{array}{l}2 / 12 / 96 \\
11 / 4 / 95\end{array}$ \\
\hline 06478540 & $\begin{array}{l}\text { Little Vermillion River near } \\
\text { Salem }\end{array}$ & 1967-97 & 78.6 & 10.01 & 1,560 & $3 / 28$ & $10-25$ & 11.95 & 3,300 & $7 / 4 / 93$ \\
\hline 06478600 & $\begin{array}{l}\text { East Fork Vermillion River } \\
\text { near Parker }\end{array}$ & 1996-97 & 973 & $\begin{array}{l}12.75 \\
12.73\end{array}$ & $\begin{array}{r}\text { BW } \\
4,210\end{array}$ & $\begin{array}{l}3 / 22 \\
3 / 29\end{array}$ & -- & & 1,380 & $6 / 3 / 96$ \\
\hline 06479000 & $\begin{array}{l}\text { Vermillion River near } \\
\text { Wakonda }\end{array}$ & 1946-97 & 1,676 & 16.93 & 6,150 & $3 / 30$ & $10-25$ & 17.62 & 17,000 & $6 / 23 / 84$ \\
\hline 06479010 & $\begin{array}{l}\text { Vermillion River near } \\
\text { Vermillion }\end{array}$ & 1984-97 & 1,808 & 22.10 & 6,070 & $4 / 2$ & $5-10$ & & 21,400 & $6 / 23 / 84$ \\
\hline 06479215 & Big Sioux River near Florence & 1984-97 & 68 & $\begin{array}{l}9.52 \\
9.32\end{array}$ & $\begin{array}{r}\mathrm{BW} \\
2,000\end{array}$ & $\begin{array}{l}4 / 2 \\
4 / 4\end{array}$ & $10-25$ & $\begin{array}{l}9.18 \\
9.08\end{array}$ & $\begin{array}{l}1,280 \\
1,810\end{array}$ & $\begin{array}{l}7 / 25 / 93 \\
3 / 29 / 86\end{array}$ \\
\hline 06479430 & $\begin{array}{l}\text { Still Lake Outflow near } \\
\text { Florence }\end{array}$ & 1996-97 & -- & $\begin{array}{r}7.43 \\
{ }^{4} 6.67\end{array}$ & $\begin{array}{r}\text { BW } \\
{ }^{4} 408\end{array}$ & $\begin{array}{l}4 / 7 \\
4 / 19\end{array}$ & -- & & & \\
\hline 06479438 & $\begin{array}{l}\text { Big Sioux River near } \\
\text { Watertown }\end{array}$ & 1973-97 & 228 & 12.09 & 7,820 & $4 / 5$ & $25-50$ & $\begin{array}{l}11.13 \\
11.08\end{array}$ & $\begin{array}{r}\text { BW } \\
4,970\end{array}$ & $\begin{array}{l}6 / 21 / 91 \\
3 / 30 / 86\end{array}$ \\
\hline 06479450 & $\begin{array}{l}\text { Lake Kampeska inlet/outlet } \\
\text { near Watertown }\end{array}$ & 1994-97 & 28.8 & $\begin{array}{r}25.78 \\
{ }^{4} 24.87\end{array}$ & $\begin{array}{r}5,890 \\
41,410\end{array}$ & $\begin{array}{l}4 / 6 \text { int } \\
4 / 10 \mathrm{or}\end{array}$ & $\begin{array}{l}\text { lake } \\
\text { t of lake -- }\end{array}$ & & -- & -- \\
\hline 06479500 & Big Sioux River at Watertown & $\begin{array}{l}1946-72 \\
1997\end{array}$ & 350 & 12.49 & 5,800 & $4 / 6$ & $>500$ & $\begin{array}{l}11.40 \\
10.30\end{array}$ & $\begin{array}{r}\text { BW } \\
2,220\end{array}$ & $\begin{array}{l}4 / 8 / 69 \\
4 / 9 / 52\end{array}$ \\
\hline 06479515 & Willow Creek near Watertown & & 110 & 10.93 & 3,650 & $4 / 5$ & $10-25$ & & $\begin{array}{r}\text { BW } \\
4,040\end{array}$ & $\begin{array}{l}3 / 28 / 78 \\
6 / 15 / 84\end{array}$ \\
\hline 06479520 & $\begin{array}{l}\text { Big Sioux River below } \\
\text { Watertown }\end{array}$ & 1995-97 & 511 & $\begin{array}{l}13.13 \\
12.99\end{array}$ & $\begin{array}{r}\mathrm{BW} \\
6,300\end{array}$ & $\begin{array}{l}4 / 2 \\
4 / 11\end{array}$ & -- & $\begin{array}{l}11.78 \\
11.74\end{array}$ & $\begin{array}{r}\mathrm{BW} \\
21,600\end{array}$ & $\begin{array}{l}3 / 13 / 96 \\
3 / 12 / 95\end{array}$ \\
\hline 06479525 & $\begin{array}{l}\text { Big Sioux River near } \\
\text { Castlewood }\end{array}$ & $1977-97$ & 570 & $\begin{array}{l}13.19 \\
12.87\end{array}$ & $\begin{array}{r}\text { BW } \\
24,300\end{array}$ & $\begin{array}{l}4 / 7 \\
4 / 11\end{array}$ & $>100$ & & 2,250 & $3 / 30 / 86$ \\
\hline 06479980 & Medary Creek near Brookings & 1981-97 & 200 & 13.02 & 23,500 & $3 / 28$ & $10-25$ & 11.78 & 3,710 & $7 / 4 / 93$ \\
\hline 06480000 & $\begin{array}{l}\text { Big Sioux River near } \\
\text { Brookings }\end{array}$ & 1954-97 & 2,419 & $\begin{array}{l}13.29 \\
13.02\end{array}$ & $\begin{array}{r}\text { BW } \\
11,100\end{array}$ & $\begin{array}{l}4 / 8 \\
4 / 2\end{array}$ & $10-25$ & & 33,900 & $4 / 9 / 69$ \\
\hline 06481000 & $\begin{array}{l}\text { Big Sioux River near Dell } \\
\text { Rapids }\end{array}$ & 1949-97 & 3,004 & 15.54 & 16,500 & $4 / 7$ & $10-25$ & 16.47 & 41,300 & $4 / 9 / 69$ \\
\hline 06482020 & $\begin{array}{l}\text { Big Sioux River at North Cliff } \\
\text { Avenue at Sioux Falls }\end{array}$ & $1972-97$ & 3,729 & 23.11 & 17,700 & $4 / 7$ & $25-50$ & & 21,600 & $6 / 22 / 84$ \\
\hline 06485500 & Big Sioux River at Akron, IA & 1929-97 & 6,937 & 22.63 & 31,300 & $4 / 9$ & $5-10$ & $\begin{array}{l}23.05 \\
22.99\end{array}$ & $\begin{array}{l}66,700 \\
80,800\end{array}$ & $\begin{array}{r}5 / 10 / 93 \\
4 / 9 / 69\end{array}$ \\
\hline
\end{tabular}

\footnotetext{
${ }^{1}$ Recurrence intervals given for stations with 10 or more years of record through 1996

${ }^{2}$ Stage-flow relation affected by backwater.

${ }^{3}$ Site and datum then in use.

${ }^{4}$ Mean daily.
} 
filled ice-covered river channels. The Grand and Moreau Rivers experienced lowland flooding from high flows and backwater from ice jams. The Moreau River near Whitehorse had a record flow of $29,700 \mathrm{ft}^{3} / \mathrm{s}$ (cubic feet per second) and record stage of $27.68 \mathrm{ft}$ (feet), as shown in table 1. Another record occurred on Oak Creek, which peaked at $19.62 \mathrm{ft}$, inundating most of the community of Wakpala.

When snowmelt began in late March in the eastern part of the State, ice thickness on rivers exceeded $3 \mathrm{ft}$. At the city of Westport in the James River Basin, heavy accumulations of ice created jams causing backwater on the Elm River that forced evacuation of several neighborhoods and caused the river to spill into the Moccasin Creek Basin, which then affected the city of Aberdeen. Snake Creek near Ashton had a recorded peak flow with a recurrence interval greater than 100 years. Tributary flows entering the main stem of the James River caused extreme backwater from Columbia to near Redfield. Record reverse flows were measured on the James River at Columbia $\left(-2,430 \mathrm{ft}^{3} / \mathrm{s}\right.$ on March 30) and at Ashton $\left(-8,400 \mathrm{ft}^{3} / \mathrm{s}\right.$ on March 31$)$, when excessive tributary inflows flowed upstream in the river. Accumulating downstream inflows caused evacuation of James Valley Christian School when dikes failed on April 6 and the school was inundated with 5-7 ft of flood water. The James River at Huron rose to a record stage of $21.28 \mathrm{ft}$ with a corresponding peak flow of $23,400 \mathrm{ft}^{3} / \mathrm{s}$ on April 6, exceeding the 500-year recurrence interval. Peak-flow recurrence intervals downstream on the James River ranged from greater than 100 years at Forestburg to greater than 50 years at Scotland.

Additional severe flooding occurred in April as the heavy snowpack began to melt in the northeastern part of the State. The most adversely affected area was the upper Big Sioux River Basin near Watertown and the surrounding glacial-lakes area. The record levels reached by the Big Sioux River exceeded the 500-year recurrence interval at Watertown and forced the evacuation of many low-lying neighborhoods. Lake Kampeska residents suffered extensive damage to shoreline properties from record-high lake levels, which were caused by inflows from the Big Sioux River.

Residual soil moisture and high base flows during 1993-96, coupled with the large winter 1996-97 snow accumulations, resulted in record total flows throughout eastern South Dakota for water year 1997. The total flow for water year 1997 was 17 times the long-term median streamflow at the James River near Scotland gaging station (fig. 2).

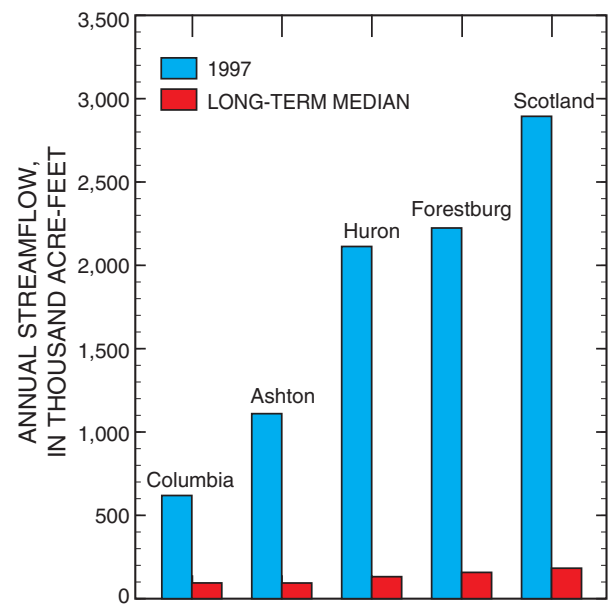

Figure 2. Comparison of 1997 streamflow to long-term median annual streamflow at selected James River gaging stations.

\section{MONITORING OF STREAMFLOW}

The USGS operates a network of 123 continuous-record streamflowgaging stations and 23 high-flow partialrecord gages in South Dakota. Miscellaneous annual streamflow measurements also are made at several additional sites throughout the State.

The data for about 50 of the continuous-record stations are relayed by satellite telemetry to computers in Rapid City, Huron, and Pierre. Data are transmitted every 4 hours, and within 15 minutes these data generally are available to decision makers in the agencies involved in flood management. In addition, data from selected stations within various river basins are displayed via the South Dakota District Homepage on the World Wide Web at: http://wwwsd.cr.usgs.gov. The flood-tracking page that was maintained during the extreme 1997 flooding for the James River Basin is shown in figure 3.
This flood-tracking page, and similar ones for the Vermillion and Big Sioux Basins, made timely data readily accessible to the public, media, and cooperating agencies. The USGS and cooperating agencies also operate a network of telephone-accessible gages where critical stage data are available on a real-time basis.

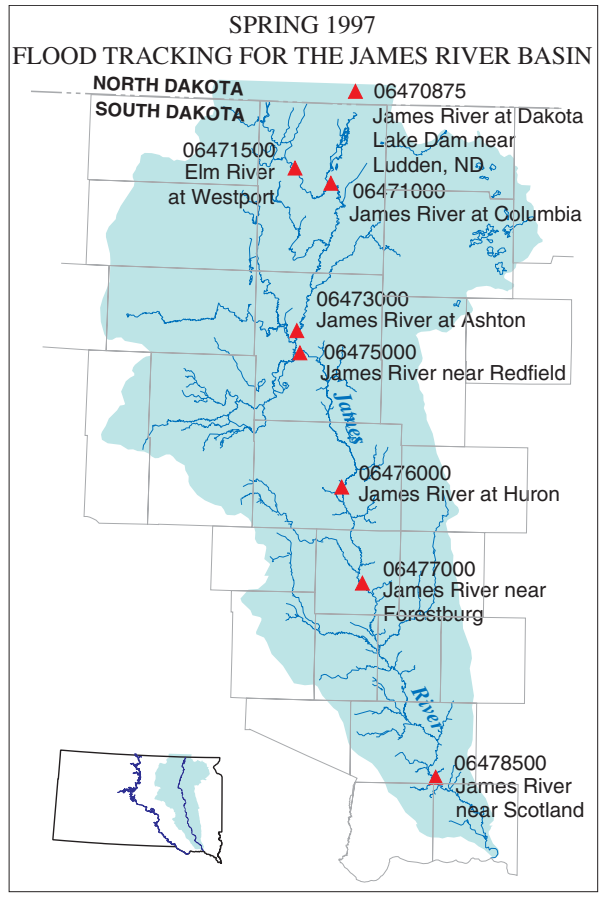

Figure 3. Flood-tracking page.

Rainfall and streamflow data in this report are provisional and subject to revision upon further review by personnel of the National Weather Service and the USGS.

-R.W. Teller and M.J.Burr

\section{For More Information:}

For water information:

District Chief

1608 Mt. View Road

Rapid City, SD 57702

(605) 355-4560

Fax: (605) 355-4523

Email: dc_sd@usgs.gov

Additional earth science information can be found by accessing the USGS Home

Page on the World Wide Web a http://www.usgs.gov

For more information on all USGS reports and products (including maps, images, and computerized data), call 1-800-USA-MAPS 